The Physics Educator

Vol. 2, No. 1 (2020) 2050001 (11 pages)

(c) The Author(s)

DOI: $10.1142 / \mathrm{S} 2661339520500018$

\title{
An Overview of the CERN Beamline for Schools Competition
}

\author{
Sarah Aretz*, Cristóvão Beirão da Cruz e Silva ${ }^{\dagger}$ and Markus Joos ${ }^{\ddagger}$ \\ CERN, Esplanade des Particules 1, \\ 1211 Geneva 23, Switzerland \\ *Sarah.Aretz@cern.ch \\ ${ }^{\dagger}$ Cristovao.bcs@cern.ch \\ ${ }^{\ddagger}$ Markus.Joos@cern.ch \\ Paul Schütze ${ }^{\S}$ and Marcel Stanitzki ${ }^{\Uparrow}$ \\ DESY, Notkestraße 85, 22607 Hamburg, Germany \\ §aul.Schuetze@desy.de \\ \Marcel.Stanitzki@desy.de
}

Received January 28, 2020; Accepted February 11, 2020

\begin{abstract}
Since 2014 CERN has been organizing "Beamline for Schools" (BL4S), an international science competition for teams of high school students. The students are asked to propose an experiment that can be realized at a particle beam line. Experienced scientists evaluate the proposals and select two winning teams that are then invited to perform their experiments with the help of professional scientists. This paper provides a brief overview of the competition and presents in more detail the conditions of the beam lines as well as the pool of detectors and instruments that are available to the students. The paper closes with an outlook on the future of the competition.
\end{abstract}

Keywords: CERN; DESY; BL4S; high school students; particle physics; science competition.

\section{Introduction}

When CERN in 2014 celebrated its 60th anniversary it took the initiative to allow high school students to propose particle physics experiments for one of its beam lines. With this, Beamline for Schools (BL4S) was born.12 Since the beginning of the BL4S program, the goal was to create - to the extent possible - the same conditions for high school students as those which apply to the professional researchers that request beam time at CERN. This means that the students have to come up with a scientific question or a detector development project themselves, have to acquire the necessary skills and knowledge, write down their proposal in a structured way and, in case they win the competition, make a sizeable contribution to the actual execution of the experiment and the analysis of the data. While this may sound very demanding for high-school students that may not have had any classes in particle physics, the experience from the last six years has shown that the concept works very well and has a large potential to motivate students to perform extracurricular work. So far, more than 1000 teams representing about 10000 students from

This is an Open Access article published by World Scientific Publishing Company. It is distributed under the terms of the Creative Commons Attribution 4.0 (CC BY) License which permits use, distribution and reproduction in any medium, provided the original work is properly cited. 


\section{BL4S Applications 2014-2019}

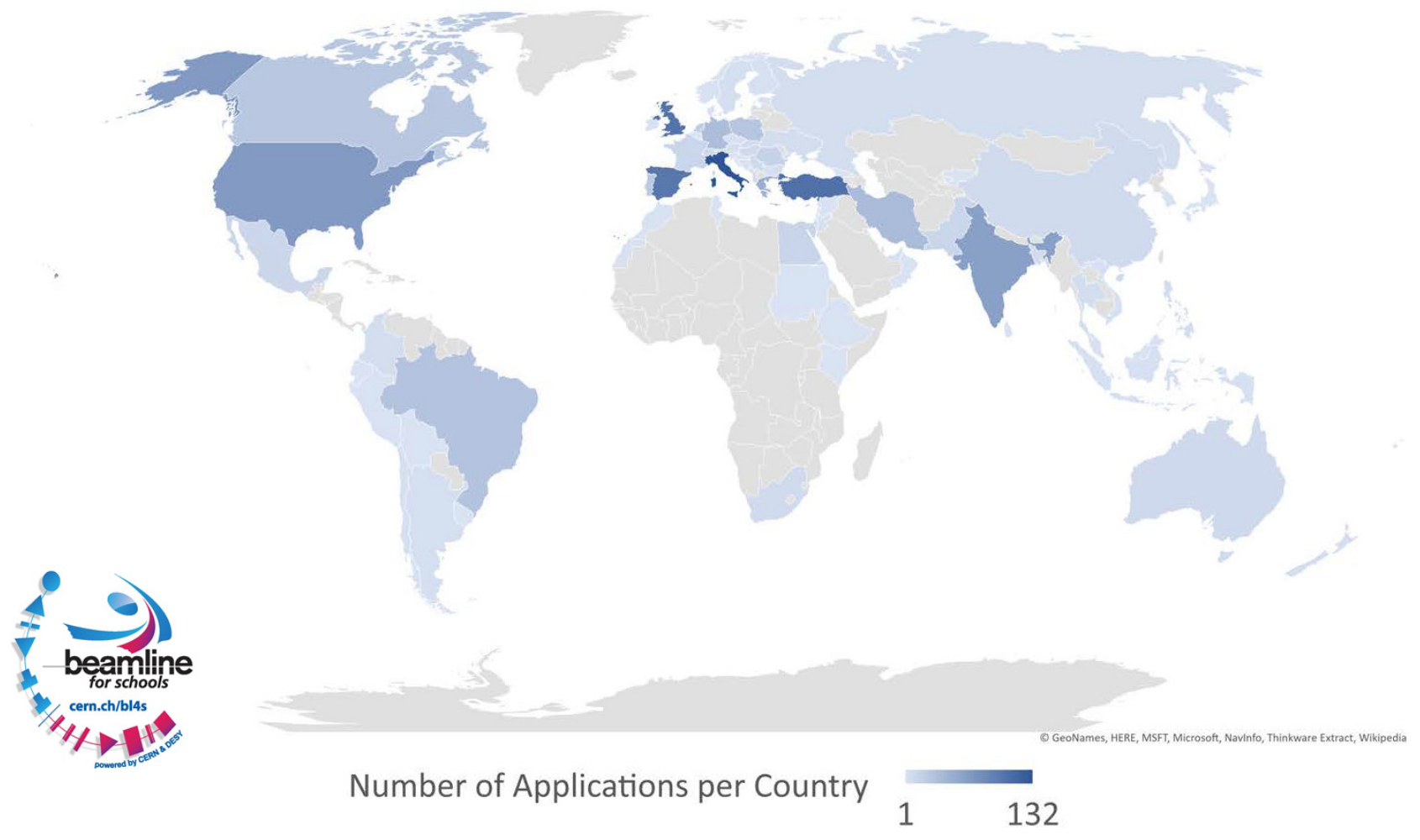

Fig. 1. Overview of the teams participating in BL4S since 2014.

84 countries worldwide have participated in BL4S (see Fig. 1).

Each team of students must be represented by an adult supervisor, the so-called team coach. Very frequently this team coach also acts as their mentor and can but does not have to be a teacher. Some teachers use BL4S as the basis for a compulsory physics project or to motivate part of their students to look deeper into modern physics, aiming to prepare them better for university or to bring them in contact with additional career perspectives.

However, in many cases the students make progress without the help of a teacher. Some teams organize themselves and the students contribute according to their skills and interest. BL4S helps them by providing a list of national contacts of professional physicists, who voluntarily mentor the teams and answer their questions during their proposal development. Some of the teams also make contact with professors at a local university. There have even been multinational teams that organized themselves via social media. Thus, even if teachers cannot coach them in person, they can help their students to participate by giving them the time, the encouragement and the environment to do so.
For teams that want to enter the competition, BL4S has prepared a document ${ }^{3}$ that describes the technical boundary conditions with respect to the features of the beam lines, at which the experiments will take place, and the detectors, electronics and software that are available to the students. The key points of this document are summarized in the following sections.

While learning about the beam features and instrumentation, the students get in touch with some basic concepts and terminology of particle physics and may have to consult further literature or experts in order to develop a deeper understanding of these. Their next and main challenge will be to formulate a research question. We know from many teams that ideas come in easily, but often the students realize that their first idea is not feasible within the conditions of BL4S. Some teams have evaluated and abandoned many ideas until they were converging on their final proposal. In this phase, they often seek the assistance from teachers, the national contacts or professors at their local university. In addition, BL4S also provides a document with feasible example experiments, $\frac{14}{4}$ which students can work out in more detail. Finally, the team has 
to write up the proposal and complement it by a short one-minute video. Feedback from the student surveys has shown that the teams spend on average about 35 hours on the preparation of their proposal.

\section{The Beam Lines}

For the first five years (2014-2018), the BL4S experiments took place at the Proton Synchrotron (PS) of CERN ${ }^{[5}$ In 2019 and 2020, the entire complex of accelerators at CERN is shut down for maintenance and upgrade. In order to continue BL4S, a collaboration with DESY in Hamburg, Germany has been set up, such that the winning teams perform their experiments at the DESY II Test Beam Facility ${ }^{[}$

\subsection{CERN PS beam test areas}

Built in 1959 the PS still plays a pivotal role in the complex of accelerators at CERN $\stackrel{5}{5}$ In addition to pre-accelerating particles for the SPS and LHC it provides particles for fixed target experiments in a facility called the East Hall, where in total four beam lines are available. BL4S uses the beam line called $T 9$, which is described below and shown in Fig. 2. At the end of 2018 the beam line was closed and is currently being refurbished. When resuming operation in 2021, some of the parameters are anticipated to slightly change, which is outlined in Sec. 7 .

The proton beam is extracted from the PS with a momentum of $24 \mathrm{GeV} / \mathrm{c}$ and hits a target, producing a beam of secondary particles. Bending magnets and a collimator, all fully controllable by the users, determine the properties of the secondary beam. The bending magnets are used in the beam line to choose the momenta of the secondary particles in the range between $0.5 \mathrm{GeV} / \mathrm{c}$ and $10 \mathrm{GeV} / \mathrm{c}$.

There are two collimators available in the T9 beam line. The horizontal and vertical collimators filter particles according to the angle of their trajectory. The horizontal collimator changes the width of the momentum distribution, depending on its opening. Thus, it rejects particles that have either a higher or a lower momentum than a predetermined range. Afterwards the beam enters the permanently installed Cherenkov detectors that can perform a first particle identification measurement. A sketch of the beam line is shown in the left part of Fig. 3

The beam composition depends on the target, the selected momentum and the polarity. The negative (positive) beam contains negatively (positively) charged particles: electrons (positrons), antiprotons

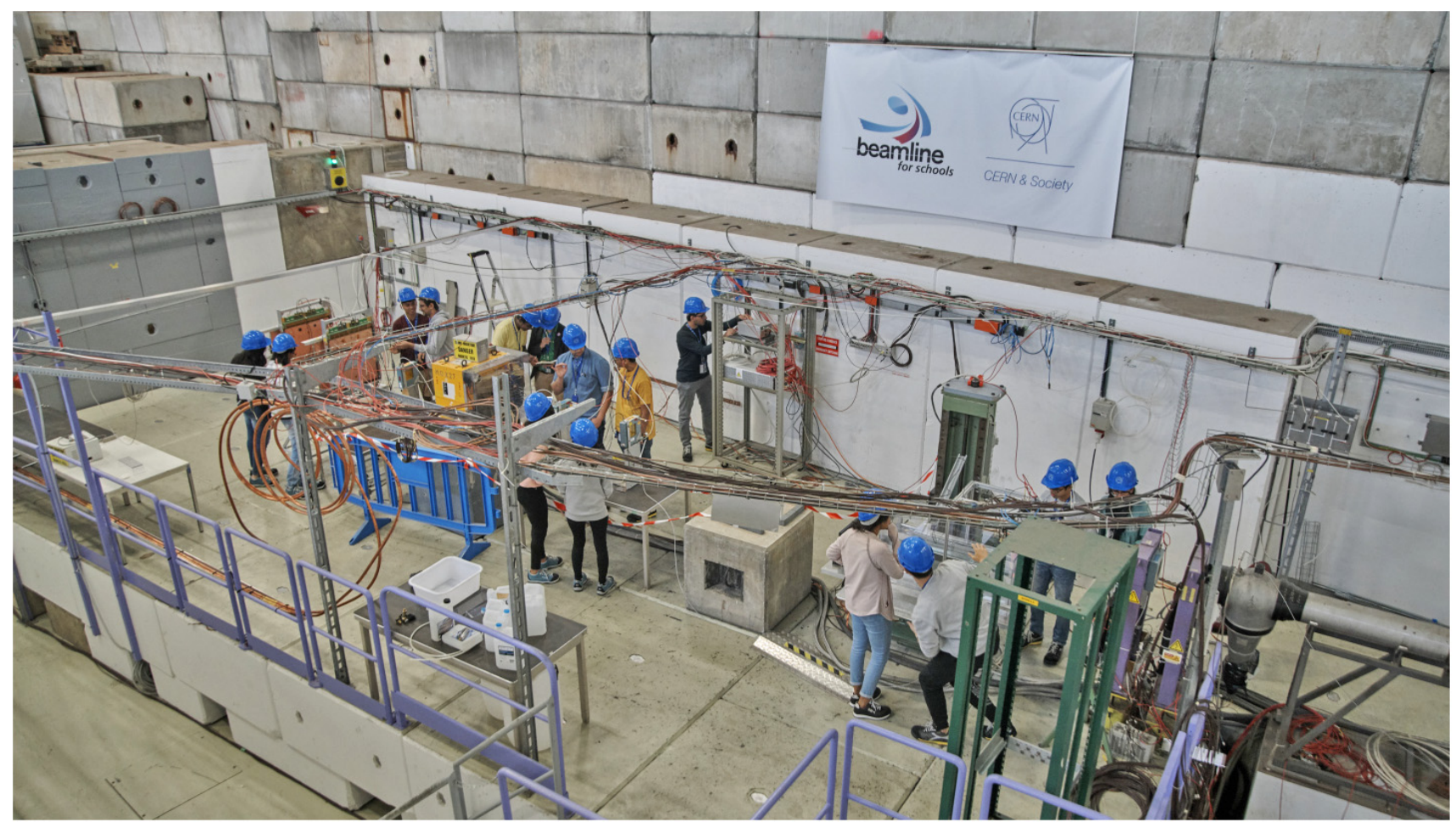

Fig. 2. Winning students of 2018 setting up their experiment in the T9 beam area at CERN (Image Copyright: CERN, Julien Marius Ordan/Rachel Tessa Lavy). 


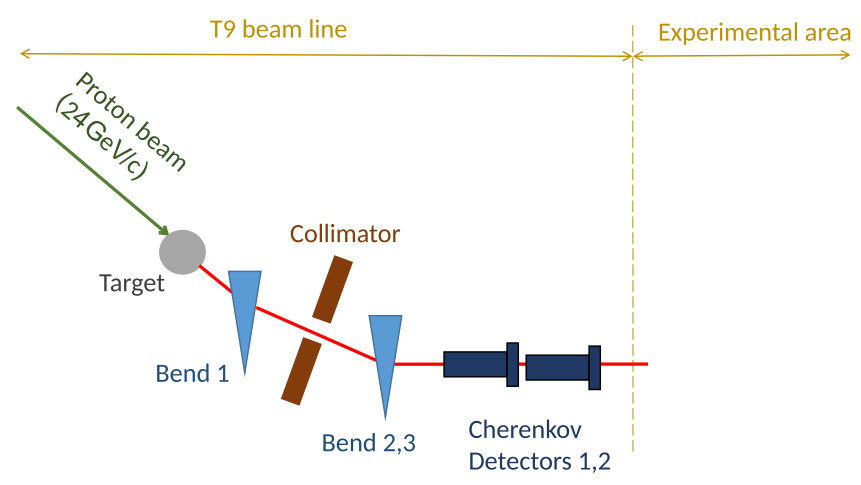

Fig. 3. Sketch of the PS T9 beam line layout. A proton beam is used to create secondary particle beams. Detailed information on the various steps along the beam path are given in the text.

(protons), pions and kaons. Figures 4 and 5 show the composition of the negative and positive beams respectively. Along their path to the experimental area, some of the pions and kaons decay, therefore there is a large number of muons entering T9. Due to the beam line configuration, it is not possible to have a beam of neutral particles. Owing to the acceleration cycle of the PS, the particles arrive in bursts, which are also called spills: about one million particles arrive throughout a $0.4 \mathrm{~s}$ long burst, at a frequency of two bursts per $40 \mathrm{~s}$. As an example, if the magnets are set up to deliver a negative beam with a momentum of $4 \mathrm{GeV} / \mathrm{c}$, each spill contains on average around $\sim 450$ antiprotons, $\sim 10000$ electrons, $\sim 10000$ kaons and $\sim 150000$ pions.

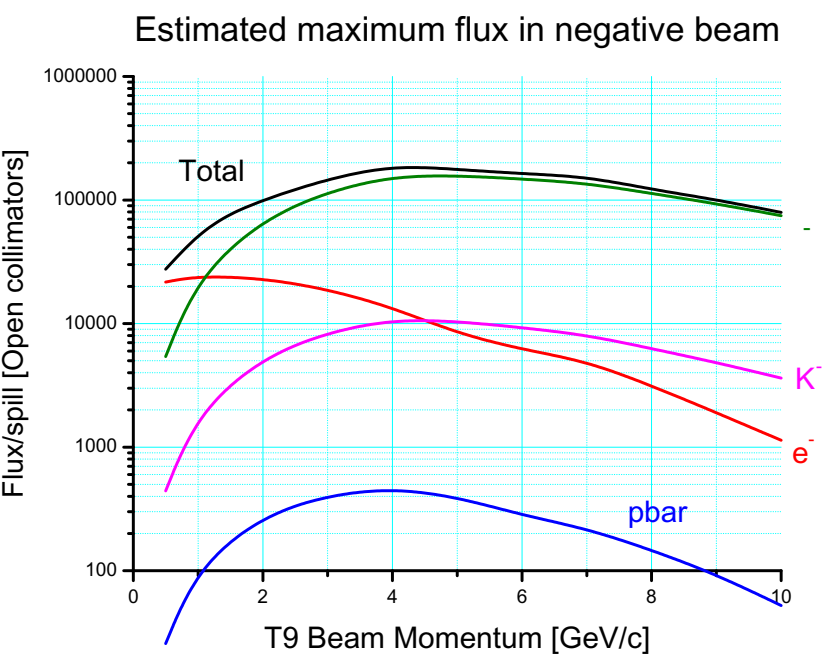

Fig. 4. Beam composition of the negative beam at the T9 beam line. Flux/spill describes the number of particles per burst. The graph labeled pbar describes the flux of antiprotons. There are no muons present at this stage, but they appear when pions or kaons decay?

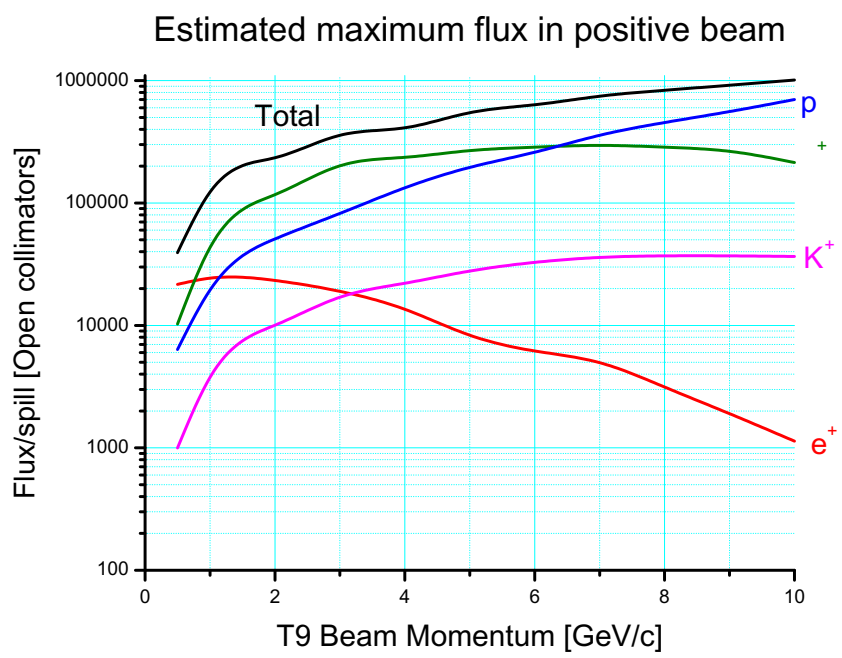

Fig. 5. Beam composition of the positive beam at the T9 beam line. Flux/spill describes the number of particles per burst. As for the positive beam, there are no muons present right after the target, but they appear when pions or kaons decay!

The beam has a more or less round, or 2D Gaussian cross-section. In the focal plane, the beam spot has a diameter of about $2 \mathrm{~cm}$. The experiments of the winning teams are installed in an area of about $5 \mathrm{~m} \times 12 \mathrm{~m}$.

\subsection{The DESY II test beam facility}

The DESY II Test Beam Facility 6 , simultaneously delivers particle beams to users at three independent beam lines called TB21, TB22 and TB24. The generation of one individual particle beam is illustrated in Fig. 6. Bremsstrahlung photons are produced when the DESY II beam is hitting the primary targets, which consist of $7 \mu \mathrm{m}$ thick carbon fibres. The photons propagate towards a secondary target where electron/positron pairs are produced in converter targets of a few millimetres thickness. These particles pass a dipole magnet with a collimator behind it at a small angle. By altering the magnetic field in the dipole and the collimator opening, the following parameters can be chosen:

- electron or positron beam,

- momentum of the beam,

- momentum spread of the beam,

- transverse profile of the beam.

The momentum can be set to any value between 1 and $6 \mathrm{GeV} / \mathrm{c}$. The amount of particles in the beam depends on the primary beam current in 


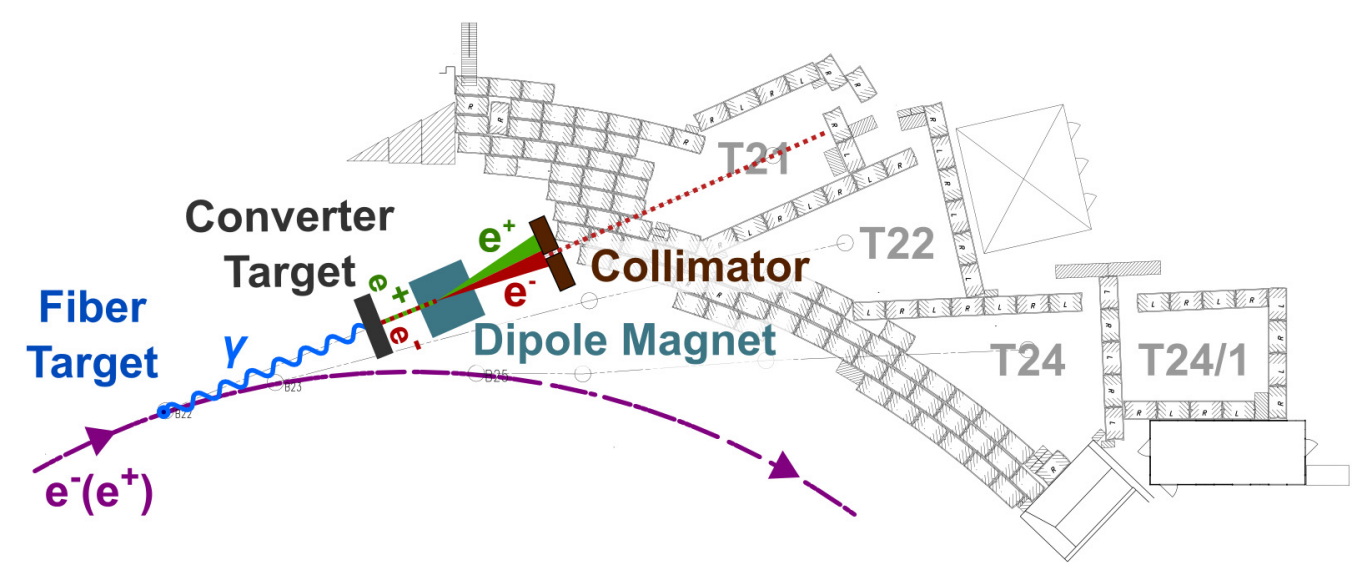

Fig. 6. Sketch of the beam generation at the DESY II Test Beam Facility. Modified from Ref. 6

DESY II, the selected momentum and the collimator opening. A typical particle rate at a beam momentum of $2 \mathrm{GeV} / \mathrm{c}$ is in the order of $10 \mathrm{kHz}$. Figure 7 shows the dependence of the particle rate

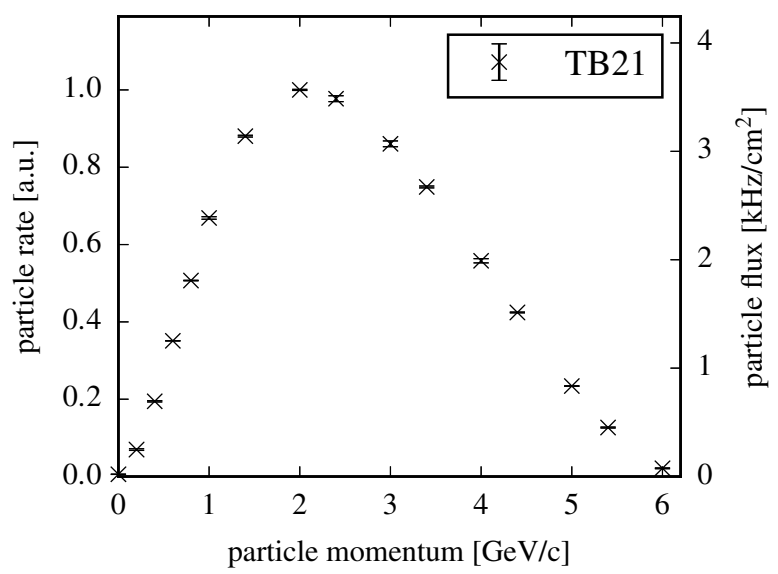

Fig. 7. Dependence of the particle rate on the selected momentum, measured in area TB21. The rate has been normalized $[$ ]

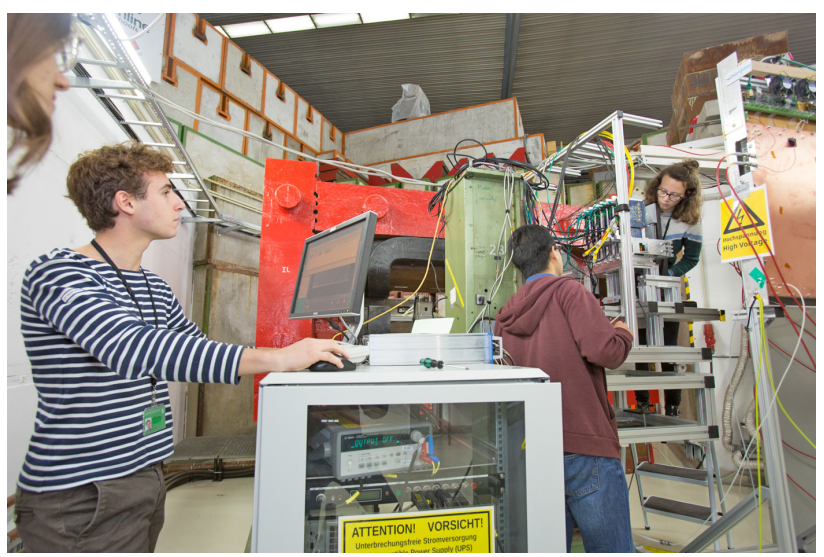

Fig. 8. Winning students of 2019 working in the DESY test beam area (Image copyright: DESY/Marta Meyer). on the selected particle momentum. For instance, at a beam momentum of $1 \mathrm{GeV} / \mathrm{c}$ the particle rate is reduced to 5000 particles per second.

As the beam line provides mostly one particle at a time, it lends itself well to experiments focusing on effects that can be studied with individual particles, such as experiments involving particle tracking.

\section{Instrumentation and Detectors}

Over the years, the BL4S team has built and collected a number of different detectors that can be used for the experiments. Further instrumentation and detectors are provided by the test beam facilities. In the following, the available equipment will be described in more detail.

\subsection{Triggering}

In most cases, scintillators are used for the purpose of triggering. We have a number of different sizes of scintillator slabs ranging from very small ones with a dimension of $1 \mathrm{~cm} \times 1 \mathrm{~cm}$ to large ones that cover an area of $25 \mathrm{~cm} \times 30 \mathrm{~cm}$. In addition, scintillator slabs with a central hole are available for use as a trigger veto. All of these scintillators are read out using large area (40 mm diameter) Photo Multiplier Tubes (PMTs).

\subsection{Tracking}

There are several detectors available for tracking purposes.

So far, in every year BL4S has used Delay Wire Chambers (DWCs, see Fig. 9), that are provided on loan by the Beam Instrumentation group of the Beams Department at CERN 9,10 


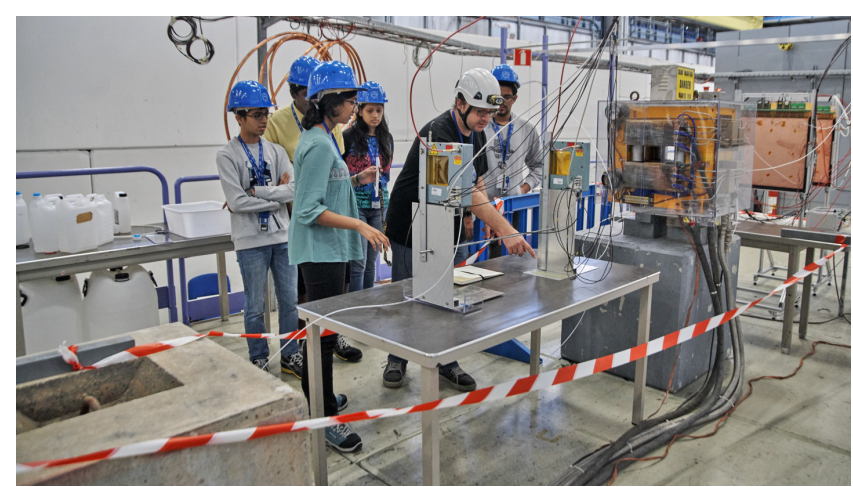

Fig. 9. Winning students and support scientists of 2018 setting up the DWCs (Image Copyright: CERN, Julien Marius Ordan/Rachel Tessa Lavy).

A DWC is a multiwire chamber from which the position of a traversing charged particle can be obtained. It uses an array of wires at high voltage connected to a delay line. The chamber is filled with a mixture of Argon and $\mathrm{CO}_{2}$. Any ionizing particle that passes through the chamber will ionize the gas. The resulting ions and electrons are accelerated by an electric field across the chamber, causing a localized cascade of ionization. The signal from the wires builds up two electric signals in the delay line, one in each direction. By using a reference signal as a common start and measuring the time delays for the signals to reach both ends of the delay line, the impact point of the original particle can be determined.

The active area is $10 \mathrm{~cm} \times 10 \mathrm{~cm}$ and position resolutions of $200 \mu \mathrm{m}$ to $300 \mu \mathrm{m}$ can be achieved. The chamber can only reliably detect one particle inside a time window of approximately $700 \mathrm{~ns}$.

In 2017 four MicroMegas 11 (see Fig. 10) detectors have been built for BL4S by the CERN

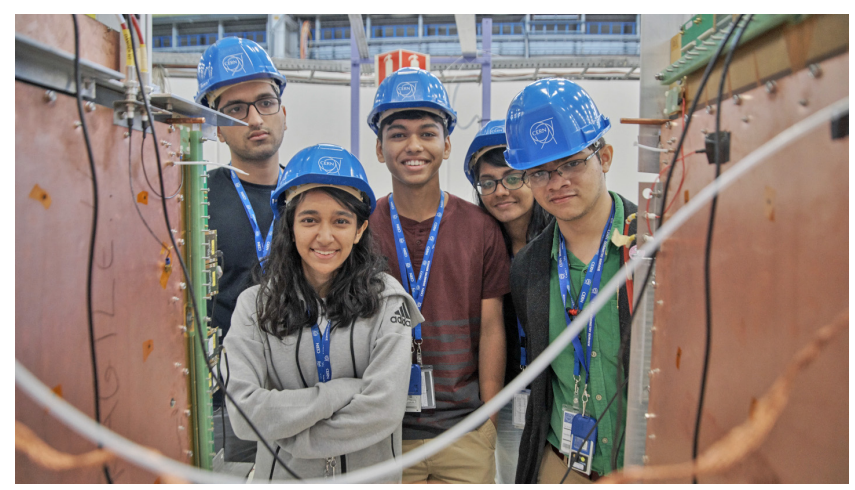

Fig. 10. Winning students of 2018 in between the MicroMegas detectors (Image Copyright: CERN, Julien Marius Ordan/Rachel Tessa Lavy). gas detector workshop, providing a spatial resolution of about $200 \mu \mathrm{m}$. The main advantage of the MicroMegas with respect to the DWCs is the larger surface of $40 \mathrm{~cm} \times 40 \mathrm{~cm}$.

The BL4S MicroMegas are 1D detectors and therefore able to record the position of a charged particle in one direction. Usually they are oriented vertically or horizontally. Combining a pair of individual detectors can thus serve as a $2 \mathrm{D}$ detector. In terms of their design, they are very similar to the detectors that have been developed for the upgrade of the ATLAS experiment, ${ }^{12}$ from which BL4S benefitted by means of technical support during the construction and commissioning of these detectors. While these MicroMegas are mechanically relatively robust, it has turned out that they are very sensitive to humidity.

The third type of tracking detector available to BL4S experiments are silicon pixel detectors in the form of beam telescopes. These beam telescopes consist of typically six planes of silicon pixel detectors, subsequently ordered along the beam axis.13 14

Silicon pixel detectors detect traversing charged particles by their ionization in the sensor material and a collection of the electrons or holes at a readout electrode. ${ }^{15}$ Segmenting this readout electrode in two dimensions then yields a 2D information on the position of particle traversal. With multiple 2D detectors along the particle path, the particles' trajectories can be reconstructed.

Compared to the DWC and the MicroMegas detector, the available beam telescopes have by far the best spatial resolution and therefore provide the most accurate tracking. The disadvantage of the sensors of the telescopes is the relatively small surface of only $2 \mathrm{~cm} \times 1 \mathrm{~cm}$.

\subsection{Time of flight}

For time of flight (TOF) measurements, three Multi-gap Resistive Plate Chambers (MRPC) have been built for BL4S!16 They have a surface area of $30 \mathrm{~cm} \times 30 \mathrm{~cm}$ and, in a well-calibrated system, a time resolution as low as $100 \mathrm{ps}$ can be reached. The readout is based on V1290 time-to-digital converter (TDC) modules $\frac{17}{17}$ by CAEN, that provide a time resolution of $25 \mathrm{ps}$.

The MRPCs consist of a stack of resistive plates, where spacers between these plates define a series of gas gaps. Anode and cathode electrodes are placed on the outer surfaces of the outermost 
resistive plates while all interior plates are left electrically floating. The resistive plates are transparent to the fast signals generated by the avalanches inside each gas gap, caused by a traversing particle. The induced signal on the external electrodes is the sum of the signals of all the gaps. The main disadvantage of the MRPCs is that they have to be operated with a mixture of Freon and $\mathrm{SF}_{6}$, which are both strong greenhouse gases.

Alternatively, but with an inferior time resolution compared to the MRPC detectors, TOF measurements can be realized with scintillators.

\subsection{Particle identification}

In the T9 beam line at CERN, two Cherenkov detectors are available. 18 They are $2.5 \mathrm{~m}$ and $5 \mathrm{~m}$ long and can be operated with different gases up to a pressure of three bar. At a given momentum range the discrimination between electrons, muons and pions is possible by tuning the pressure of the gas. Identifying heavier particles, such as kaons or protons, is not possible with these units.

\subsection{Calorimetry}

From the OPAL experiment ${ }^{\frac{19}{19}}$ BL4S has inherited 16 lead glass calorimeters that each have a volume of $10 \mathrm{~cm} \times 10 \mathrm{~cm} \times 37 \mathrm{~cm}$. They are read out with PMTs and charge-to-digital converters (QDCs) of the type CAEN V792 $2^{20}$ and work best for particles with a momentum of more than $500 \mathrm{MeV} / \mathrm{c}$.

\subsection{Magnets}

At CERN and DESY, several dipole magnets with fields of up to $1.35 \mathrm{~T}$ are available in order to deflect charged particles, as well as a superconducting solenoid with a magnetic field of up to $1.0 \mathrm{~T}$ at DESY. In combination with the tracking detectors they allow for spectrometric measurements or for tests of detectors or electronic components in a magnetic field.

\subsection{Other detectors and equipment}

For two of the winning experiments in 2015 additional detectors - a low energy calorimeter and a Timepix sensor - were borrowed from different research groups.

The winners of BL4S may also bring their own detectors with them. In the past, winning teams have constructed a calorimeter and Cherenkov detectors at their schools.

\section{Data Acquisition System}

The readout and configuration of the available detectors demand a set of both hardware and software tools. For every experiment, the data acquisition system is set up individually, making use of existing, though customized software frameworks and the hardware available to BL4S.

\subsection{Hardware}

In order to read out the detectors, a number of different solutions based on both commercial and custom electronics modules is used. For most of the experiments, NIM modules 21 are used to set up a trigger logic. The digitization of the analog signals from scintillators, DWCs, MRPCs and calorimeters is based on both TDC and QDC VMEbus modules. These modules are in turn read out by an embedded VMEbus processor. The MicroMegas require customized readout electronics and are thus read out using electronics developed by the ATLAS collaboration, called MMFE8 (MicroMegas Front-End) cards. For the beam telescopes, a full data acquisition system is stationary at both the CERN and DESY beam lines.

\subsection{Software}

The data acquisition system of BL4S is mostly based on the RCD TDAQ framework ${ }^{[22}$ It is a modular system that has been developed more than 15

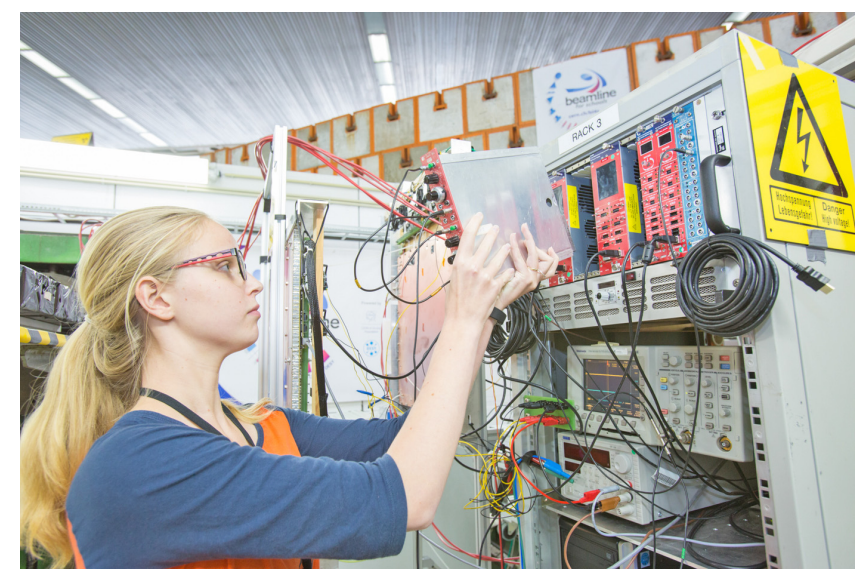

Fig. 11. Winning student of 2019 installing an electronics module in the trigger system (Image copyright: DESY/Marta Meyer). 


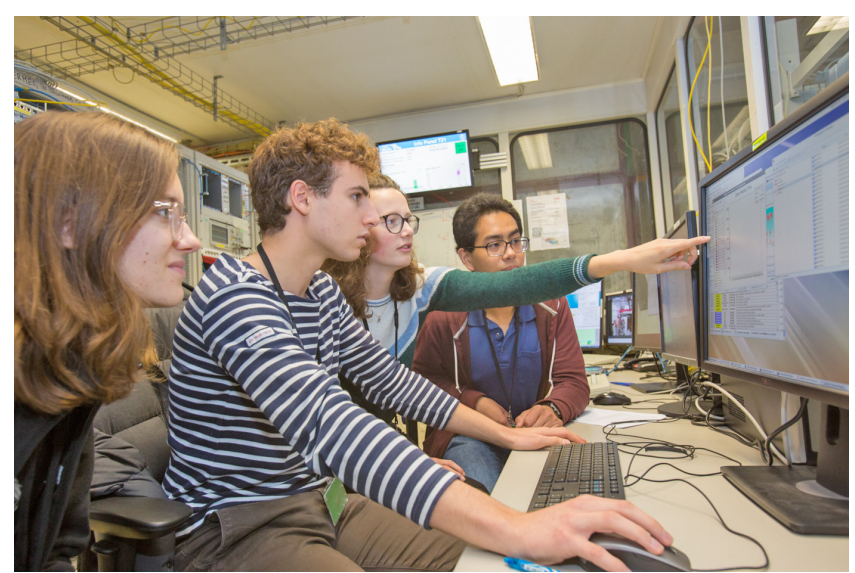

Fig. 12. Winning students of 2019 working in the control room at DESY (Image copyright: DESY/Marta Meyer).

years ago for the ATLAS experiment, to be used in both test beams and the actual experiment.

RCD TDAQ runs on a VMEbus processor and is able to cope with a data volume of $5-10 \mathrm{MB} / \mathrm{s}$. Software modules have been developed in order to receive data via network interfaces from the custom electronics of the MicroMegas and beam telescope.

The beam telescopes are configured and read out using the EUDAQ data acquisition framework $13 \underline{13}$

\section{Winning Experiments}

So far, twelve teams have won the BL4S competition. Ten teams have been invited to CERN in 2014 to 2018 and two to DESY in 2019. Table 1 summarizes the winning experiments.

\section{Sponsoring}

Starting from the very first edition, BL4S has received growing financial support from a number of donors. This third party money, raised by the CERN and Society Foundation, covers the expenses for the invitation of the winners, prices for the short listed teams, the purchased equipment for the experiments and parts of the salaries of the support scientists.

\section{Future of BL4S}

In the currently ongoing 2020 edition, the two winning teams will again be invited to DESY. The future of BL4S beyond 2020 is currently under discussion. CERN will again be able to host the competition at the then refurbished beam lines of the PS accelerator. The T9 beam line will in the future allow for beam energies ranging from 0.2 to $15 \mathrm{GeV} / \mathrm{c}$.24

Table 1. BL4S winning teams and their proposed experiments.

\begin{tabular}{|c|c|c|}
\hline Year & Team Name and Country & Proposal \\
\hline \multirow[t]{2}{*}{2014} & Odysseus Comrades, Greece & $\begin{array}{l}\text { Decay of pions into either a muon or an electron (and a neutrino). This was an } \\
\text { attempt to reproduce one of the first experiments done at CERN in } 1957\end{array}$ \\
\hline & Dominicus College, Netherlands & Test of several calorimeters made from self-grown crystals and commercial PMTs \\
\hline 2015 & Accelerating Africa, South Africa & $\begin{array}{l}\text { Detection of channelling radiation emitted by fast electrons in a doped artificial } \\
\text { diamond }\end{array}$ \\
\hline 2016 & $\begin{array}{l}\text { Relatively Special, UK } \\
\text { Pyramid Hunters, Poland }\end{array}$ & $\begin{array}{l}\text { Measurement of the Lorentz factor using highly relativistic pions } \\
\text { Measurement of the absorption of muons in limestone in order to better under- } \\
\text { stand the concept of muon radiography }\end{array}$ \\
\hline 2017 & $\begin{array}{l}\text { Charging Cavaliers, Canada } \\
\text { TCO-ASA, Italy }\end{array}$ & $\begin{array}{l}\text { Search for fractionally charged elementary particles } \\
\text { Construction of a Cherenkov detector from a water tank and several types of } \\
\text { light sensors }\end{array}$ \\
\hline 2019 & $\begin{array}{l}\text { DESY Chain, USA } \\
\text { Particle Peers, Netherlands }\end{array}$ & $\begin{array}{l}\text { Study of the efficiency of scintillators } \\
\text { Measurement of the shape of a particle shower in a tungsten target }\end{array}$ \\
\hline
\end{tabular}




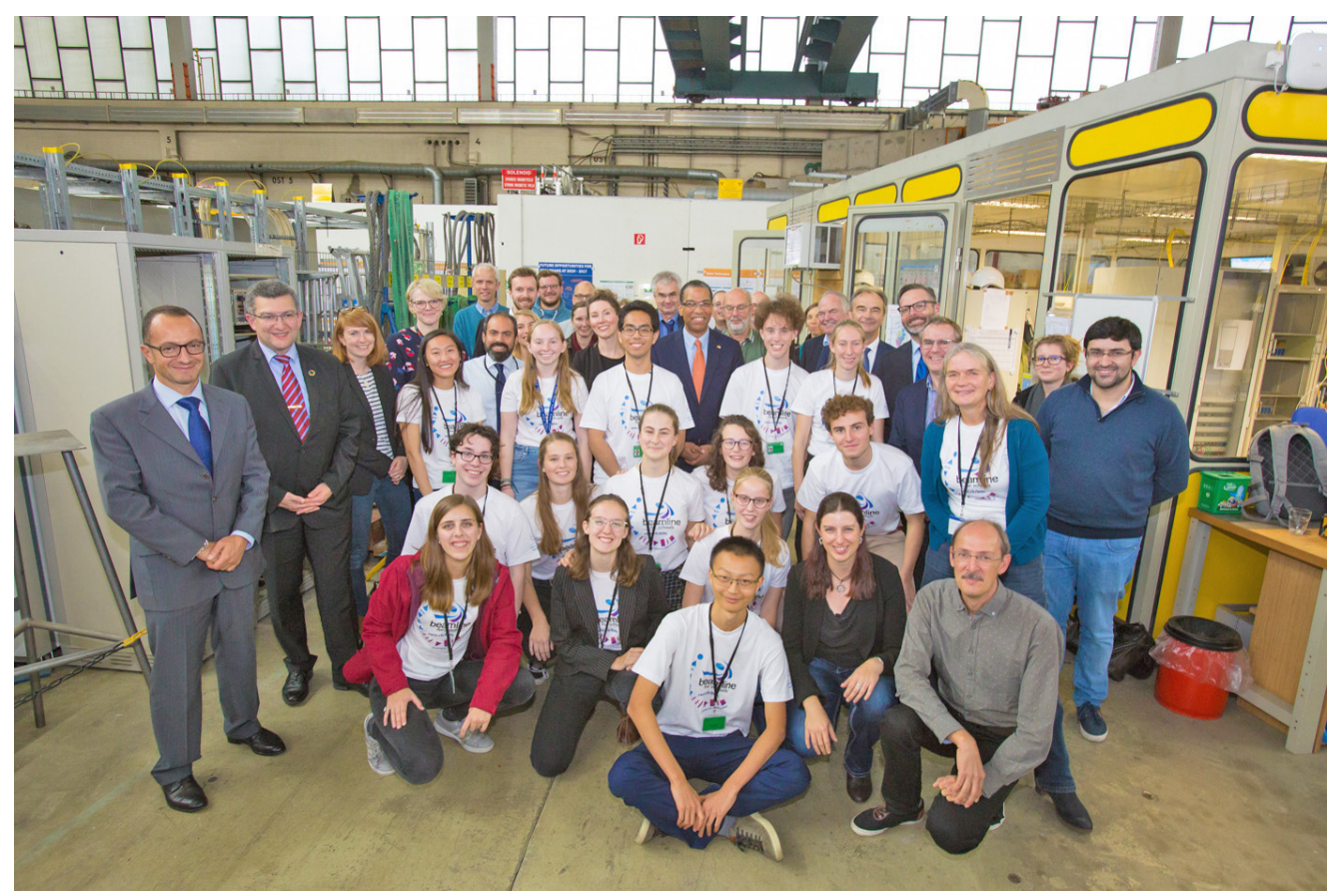

Fig. 13. The winning teams of 2019 at DESY with their teachers and officials (Image copyright: DESY/Marta Meyer).

In addition, it will be possible to get almost pure beams of electrons/positrons or hadrons. The specification of the momentum of muons is also expected to improve and the total rate of particles may approach up to $1 \mathrm{MHz}$. This turns T9 into a facility that allows for an even larger spectrum of experimental proposals.

A decision is pending, in which form the competition will be run after 2020, whereas several models are under discussion including the collaboration with further research laboratories. Hosting the winning BL4S experiments at DESY in 2019 has shown that the competition is not bound to one particular lab, as long as the hosting lab is able to provide a particle beam, work force for the organization of the event and for the technical support, and the necessary infrastructure to host the winning students.

\section{Conclusion}

Throughout the past six years, the BL4S team at CERN has created an environment that allows teams of high school students to successfully perform a large spectrum of ambitious experiments in particle physics and detector development. A set of particle detectors, either built for this purpose or on loan, has been integrated with a chain of read-out electronics, a data acquisition system and a suite of software tools for data analysis. By inviting the winning teams of the 2019 BL4S edition to DESY, it has been demonstrated that the final phase of the competition can be successfully hosted at another accelerator lab. Furthermore, it has been shown in the last six years that this international competition does not only attract many high school students from all around the world, but also provides them with insights into future career possibilities and can have a positive impact regarding an increased knowledge in particle physics and interest in STEM, the development of their social skills as well as an influence on career choices of many participating students.

\section{Acknowledgments}

Beamline for Schools is an Education and Outreach project funded by the CERN and Society Foundation and supported by individual donors, foundations and companies.

Beamline for Schools would not be possible without the dedication and hard work of teams of volunteers from CERN, DESY and other institutes. Thanks also to CERN and DESY for making their facilities available generously. 


\section{References}

1. S. Aretz and M. Joos, CERN Courier 58, 31 (2018), https://cerncourier.com/a/hands-on-education-atthe-frontiers-of-science/.

2. CERN, Beamline for Schools https://beamlinefor schools.cern, Accessed: 22.01.2020.

3. CERN and DESY, Beam and Detectors https://cds. cern.ch/record/2706829, Accessed: 22.01.2020.

4. CERN and DESY, Example for experiments that can be done at the desy beam lines https://cds. cern.ch/record/2706832, Accessed: 22.01.2020.

5. CERN, CERN PS test beam http://sba.web.cern. ch/sba/BeamsAndAreas/East/East.htm, Accessed: 22.01.2020.

6. R. Diener, J. Dreyling-Eschweiler, H. Ehrlichmann, I. Gregor, U. Kötz, U. Krämer, N. Meyners, N. Potylitsina-Kube, A. Schütz, P. Schütze and M. Stanitzki, Nucl. Instrum. Meth. Phys. Res. Sec. A: Accelerators, Spectrometers, Detectors and Associated Equipment 922, 265 (April 2019).

7. L. Gatignon, Beam composition at the CERN PS test beam area T9 Personal communication.

8. DESY, Test beams at DESY http://testbeam.desy. de, Accessed: 22.01.2020.

9. J. Spanggaard, Delay Wire Chambers - A Users Guide, Tech. Rep. SL-Note-98-023-BI, CERN (Geneva, 1998).

10. G. Charpak and F. Sauli, Nucl. Instrum. Meth. 162, 405 (1979).

11. Y. Giomataris, P. Rebourgeard, J. Robert and G. Charpak, Nucl. Instrum. Meth. Phys. Res. Sec. A: Accelerators, Spectrometers, Detectors and Associated Equipment 376, 29 (June 1996).

12. A. Zibell, J. Instrum. 9, C08013 (August 2014).

13. H. Jansen, S. Spannagel, J. Behr, A. Bulgheroni, G. Claus, E. Corrin, D. Cussans, J. DreylingEschweiler, D. Eckstein, T. Eichhorn, M. Goffe, I. M. Gregor, D. Haas, C. Muhl, H. Perrey, R. Peschke, P. Roloff, I. Rubinskiy and M. Winter, EPJ Techn. Instrum. 3 (October 2016).

14. DESY Beam Telescope Coordinators, EUDETtype beam telescopes https://telescopes.desy.de, Accessed: 22.01.2020.

15. G. Lutz, Semiconductor Radiation Detectors (Springer-Verlag, Berlin, Heidelberg, 1999).

16. E. C. Zeballos, I. Crotty, D. Hatzifotiadou, J. L. Valverde, S. Neupane, M. Williams and A. Zichichi,
Nucl. Instrum. Meth. Phys. Res. Sec. A: Accelerators, Spectrometers, Detectors and Associated Equipment 374, 132 (1996).

17. CAEN, V1290n-2esst https://www.caen.it/products/v1290n-2esst/, Accessed: 22.01.2020.

18. C. Grupen and B. Shwartz, Particle Detectors, Cambridge Monographs on Particle Physics, Nuclear Physics and Cosmology (Cambridge University Press, 2008).

19. O. Collaboration, Nucl. Instrum. Meth. Phys. Res. Sec. A: Accelerators, Spectrometers, Detectors and Associated Equipment 305, 275 (July 1991).

20. CAEN, V792 https://www.caen.it/products/v792/, Accessed: 22.01.2020.

21. U.S. Department of Energy Office of Scientific and Technical Information, Standard NIM instrumentation system https://www.osti.gov/servlets/purl/ 7120327, Accessed: 22.01.2020.

22. S. Gameiro, G. Crone, R. Ferrari, D. Francis, B. Gorini, M. Gruwé, M. Joos, G. Lehmann, L. Mapelli, A. Misiejuk, E. Pasqualucci, J. Petersen, R. Spiwoks, L. Tremblet, G. Ünel, W. R. Vandelli and Y. Yasu, The ROD Crate DAQ of the ATLAS Data Acquisition System, Tech. Rep. ATL-DAQCONF-2005-020. ATL-COM-DAQ-2005-019, CERN (Geneva, 2005).

23. P. Ahlburg, S. Arfaoui, J. H. Arling, H. Augustin, D. Barney, M. Benoit, T. Bisanz, E. Corrin, D. Cussans, D. Dannheim, J. Dreyling-Eschweiler, T. Eichhorn, A. Fiergolski, I. M. Gregor, J. GrosseKnetter, D. Haas, L. Huth, A. Irles, H. Jansen, J. Janssen, M. Keil, J. S. Keller, M. Kiehn, H. J. Kim, J. Kroll, K. Krüger, S. Kulis, J. Kvasnicka, J. Lange, Y. Liu, F. Lütticke, C. Marinas, P. Martinengo, A. Nurnberg, B. Paschen, H. Perrey, R. Peschke, D. Pitzl, D. L. Pohl, A. Quadt, T. Quast, F. Reidt, E. Rossi, I. Rubinsky, A. Rummler, H. Schreeck, P. Schütze, B. Schwenker, S. Spannagel, M. Stanitzki, U. Stolzenberg, T. Suehara, M. Suljic, G. Troska, M. Varga-Kofarago, J. Weingarten and P. Wieduwilt, Eudaq - a data acquisition software framework for common beam telescopes (2019).

24. J. Bernhard, Refurbishment of the CERN PS test beam area $\mathrm{T} 9$, personal communication.

Sarah Aretz is a physicist and educator. She has studied astrophysics at the University of Bonn in Germany and has also been a teacher for physics and mathematics in grammar school. After obtaining her Ph.D., she is now the project manager of CERN's Beamline for Schools competition since 2017. Furthermore, she is involved in physics education 
research in the Teacher and Student Programmes section of the CERN Education, Communications and Outreach group.

Cristóvão Beirão da Cruz e Silva, born in Scotland, holds a doctorate degree in physics from the "Instituto Superior Técnico" of the University of Lisbon. While working for his Ph.D., he worked in the area of experimental particle physics within the CMS collaboration. The work carried out led to him attaining his Ph.D. with distinction in 2016, for his research on the supersymmetric tau partner. In 2018, he started working with the Beamline for Schools $(B L 4 S)$ competition at CERN as a support scientist. Presently a fellow at CERN, since April 2019, he also has responsibilities in the New Small Wheel detector upgrade for the Atlas detector as well as continuing his work with the BL4S team.

Markus Joos has studied technical engineering at a University of Applied Science in Germany. In 1993, he joined CERN where he has focused on the development of software for modular electronics used in data acquisition systems. He is a co-organizer of the ISOTDAQ schools and the technical coordinator of Beamline for Schools.

Paul Schütze studied physics at the Karlsruhe Institute of Technology in Germany, where he graduated with a focus on accelerator physics and experimental particle physics. He received his Ph.D. in 2019 for his work at DESY (Hamburg, Germany) in the field of silicon pixel detectors. Continuing at DESY, he is now involved in the Beamline for Schools competition as a supporting scientist and is also a part of the support and development team at the DESY II Test Beam Facility.

Marcel Stanitzki is a physicist with a focus on detector development. He obtained his Ph.D. from the University of Karlsruhe (TH). Before joining DESY as a staff scientist, he was a postdoctoral research fellow at Yale University and a staff scientist at the STFC Rutherford Appleton Laboratory, UK. At DESY, he is responsible for the DESY II Test Beam Facility, which is hosting the Beamline for Schools Competition in 2019 and 2020. 